\title{
Delayed central nervous system superficial siderosis following brachial plexus avulsion injury
}

\author{
Report of three cases
}

\author{
Aaron A. Cohen-Gadol, M.D., William E. Krauss, M.D., and Robert J. Spinner, M.D. \\ Department of Neurologic Surgery, Mayo Clinic and Mayo Foundation, Rochester, Minnesota
}

\begin{abstract}
Chronic subarachnoid hemorrhage may cause deposition of hemosiderin on the leptomeninges and subpial layers of the neuraxis, leading to superficial siderosis (SS). The symptoms and signs of SS are progressive and fatal. Exploration of potential sites responsible for intrathecal bleeding and subsequent hemosiderin deposition may prevent disease progression. A source of hemorrhage including dural pathological entities, tumors, and vascular lesions has been previously identified in as many as 50\% of patients with SS. In this report, the authors present three patients in whom central nervous system SS developed decades after brachial plexus avulsion injury. They believe that the traumatic dural diverticula in these cases may be a potential source of bleeding. A better understanding of the pathophysiology of SS is important to develop more suitable therapies.
\end{abstract}

\section{KEY WORDS • brachial plexus injury • avulsion • superficial siderosis • pseudomeningocele}

Superficial siderosis of the central nervous system is a rare disorder resulting from chronic or repeated slow hemorrhage into the subarachnoid space that leads to hemosiderin deposition onto the cerebral and spinal leptomeninges. ${ }^{3,5-10,16,17,19}$ Approximately 100 cases of SS have been reported. ${ }^{3}$ Patients with SS exhibit a wide variety of clinical features including progressive sensorineural deafness, cerebellar ataxia, myelopathy, sphincter dysfunction, and cognitive deficits. ${ }^{3}$ Despite the advances in imaging that have facilitated earlier recognition of this condition, the exact mechanism by which hemosiderin deposition leads to neurological symptoms and signs remains elusive. ${ }^{3,9,10}$

The surgical treatment of SS depends on the identification of a bleeding source and its closure. Neoplasms or vascular malformations may be causes of recurrent bleeding. In nearly $50 \%$ of patients, the source of hemorrhage is not identified, presumably because of the very slow episodic intrathecal bleeding associated with $\mathrm{SS}^{3}$ Patients with trauma-induced dural lesions, ${ }^{2}$ including nerve root avulsion, , $, 2,4,12-15,18$ may be at an increased risk of developing SS. In this report, we describe the clinical characteristics of three patients with brachial plexus avulsion injury in whom SS developed. Two of these patients underwent surgical exploration involving their traumatic dural diverticula.

\footnotetext{
Abbreviations used in this paper: $\mathrm{BPI}=$ brachial plexus injury; $\mathrm{CSF}=$ cerebrospinal fluid; $\mathrm{MR}=$ magnetic resonance; $\mathrm{RBC}=$ red blood cell; SS = superficial siderosis.
}

\section{CASE REPORTS}

\section{Case 1}

Presentation. This 42-year-old man presented with an 8-year history of progressive bilateral weakness and incoordination of the lower extremities associated with difficulty controlling bowel and bladder function. He noted numbness below the nipple line on both sides of his body. He complained of hearing loss. He also experienced memory difficulties. Twenty-one years earlier, he had been struck by a falling heavy platform and sustained cervical spine and left brachial plexus injuries. He recovered neurological function during the following year and was left with only minimal residual weakness in his left hand.

Examination. Physical examination revealed memory deficits and moderate sensorineural hearing loss, especially in the left ear. His paraparesis was worse in his right leg. There was a T-2 sensory level apparent on pin-prick testing. All deep tendon reflexes were exaggerated and $\mathrm{Ba}-$ binski signs were present bilaterally.

Magnetic resonance imaging of the neuraxis was performed. Brain MR imaging revealed a dark rim of hypointensity around the cerebellum (especially the vermis) and spinal cord consistent with hemosiderin deposition and SS (Fig. 1). The superior cerebellar vermis was atrophic. There was focal spinal cord atrophy, and $\mathrm{T}_{2}$-weighted signal change was apparent at the C7-T2 spinal segments. Cervical spine MR imaging revealed pseudomeningoceles of the C7-T1 nerve roots due to his 

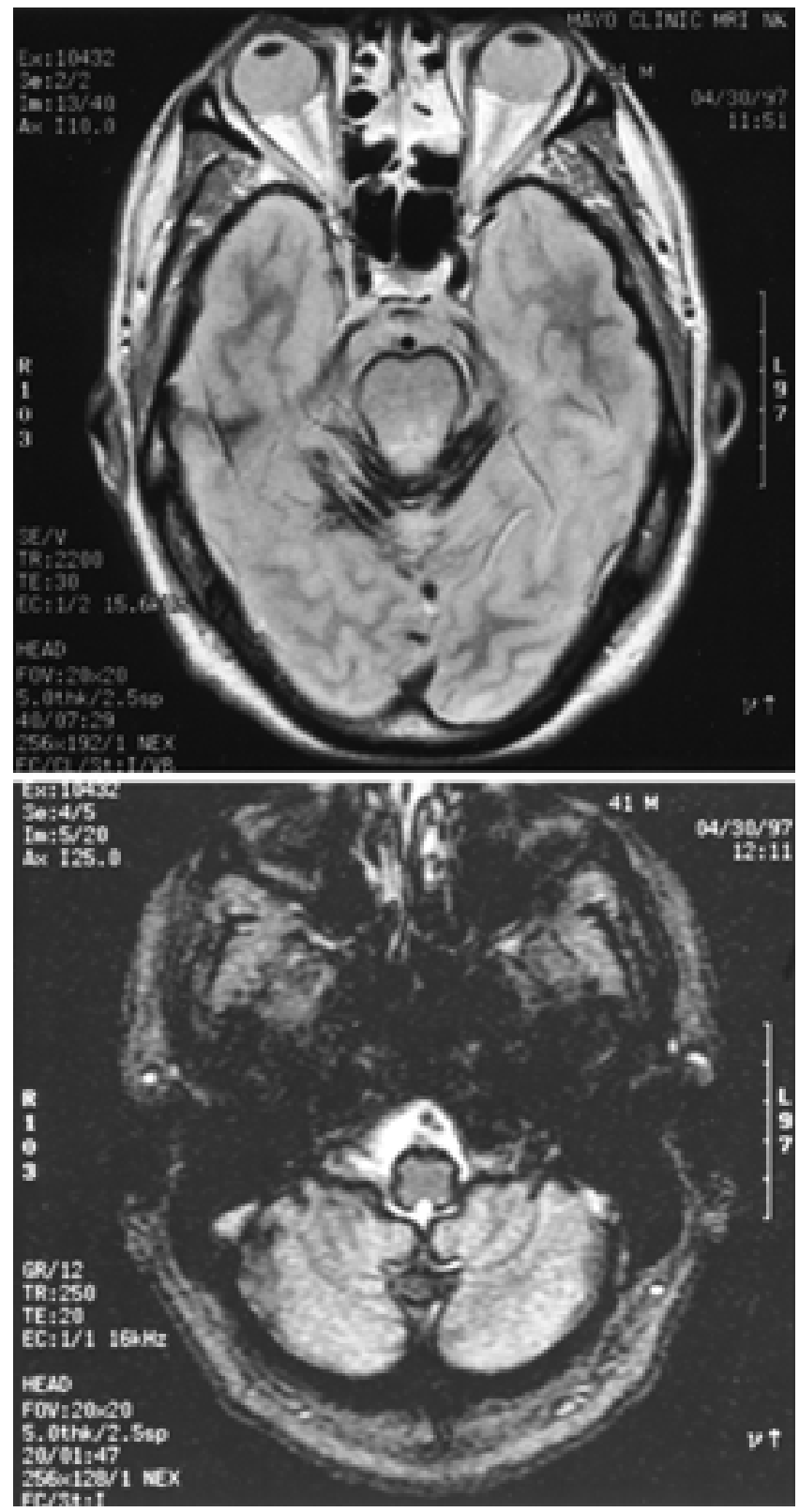

Fig. 1. Case 1. Axial proton density (upper) and gradient echo (lower) MR images demonstrating a dark rim of hypointensity around the cerebellum (especially the vermis) and brainstem consistent with hemosiderin deposition and SS.

BPI. Gadolinium-enhanced MR imaging demonstrated no additional abnormalities. Cervical myelography revealed irregular left C-8 and T-1 nerve root sleeves and a loculated anterior subdural fluid collection extending from $\mathrm{C} 7$ to T1 (Fig. 2). The dural pseudomeningoceles were considered potential sites of intrathecal hemorrhage possibly contributing to the development of SS.

Operation. We performed C7-T2 laminectomies and intradural exploration of the dural pseudomeningoceles. Friable abnormal vessels in the hyperemic scar tissue within the pseudomeningoceles were identified and coagulated. The intradural surface of the pseudomeningo- cele was disproportionately discolored compared with the rest of the spinal dura. A piece of muscle was used to occlude a small communication between the extra- and intraspinal space at the distal end of the nerve root sleeve avulsions.

Postoperative Course. This patient recovered from his surgery without event. At 2-year follow-up evaluation, no further progression of neurological deficits was noted.

\section{Case 2}

Presentation. This 52-year-old man presented with a 5year history of progressive unsteadiness, a 3-year history of hearing loss, and transient lightheadedness. He had suffered a left brachial plexus avulsion injury at 10 years of age in a farming-related accident that eliminated any function in his distal left upper limb.

Examination. Physical examination was remarkable for a broad-based gait and inability for tandem walking. He possessed functional strength in his left deltoid and biceps but none in his triceps or muscles of the wrist or hand. There was no sensation on the ulnar side of the left hand and reduced sensation on the radial side of the hand. Deep tendon reflexes were increased in the lower extremities. Toes were upgoing bilaterally.

$\mathrm{A} \mathrm{T}_{2}$-weighted brain MR imaging study revealed a rim of hypointensity around the entire neuraxis including the sylvian fissures and cerebral convexities consistent with severe SS (Fig. 3). Spinal MR imaging demonstrated similar findings as well as $\mathrm{T}_{2}$-weighted signal changes in the lower cervical spinal cord indicative of a previously unrecognized concomitant old spinal cord injury (Fig. 4 left). The rim of hypointensity around the cord was interrupted at the site of nerve root avulsion (Fig. 4 upper right). Spinal CT myelography revealed C7-T1 root sleeve avulsions with pseudomeningoceles (Fig. 4 lower right). Cerebral and spinal angiography, performed to rule out a subtle underlying vascular cause, demonstrated unremarkable findings. Preoperative CSF profile was negative for xanthochromia with only two RBCs. A radiolabeled ( $\left.{ }^{99 \mathrm{~m}} \mathrm{Tc} 21.8 \mathrm{mCi}\right) \mathrm{RBC}$ single-photon emission computerized tomography study demonstrated no site of slow intradural bleeding at 24 hours around the region of traumatic pseudomeningoceles.

Operation. We explored the pseudomeningoceles at the site of the avulsion after performing unilateral C5-T2 hemilaminectomies. The intradural surfaces of the pseudomeningoceles at C-7 to T-1 were heavily stained with hemosiderin compared with the rest of the spinal dura; however, no abnormal vessels were identified. The pseudomeningoceles were ligated and resected in an attempt to prevent future repeated hemorrhage and additional staining of neuraxis.

Postoperative Course. At the 15-month follow-up visit, the patient remained neurologically unchanged. Examination of CSF samples demonstrated no xanthochromia or RBCs.

\section{Case 3}

Presentation. This 69-year-old man presented with a 2year history of progressive imbalance during activity. $\mathrm{He}$ 


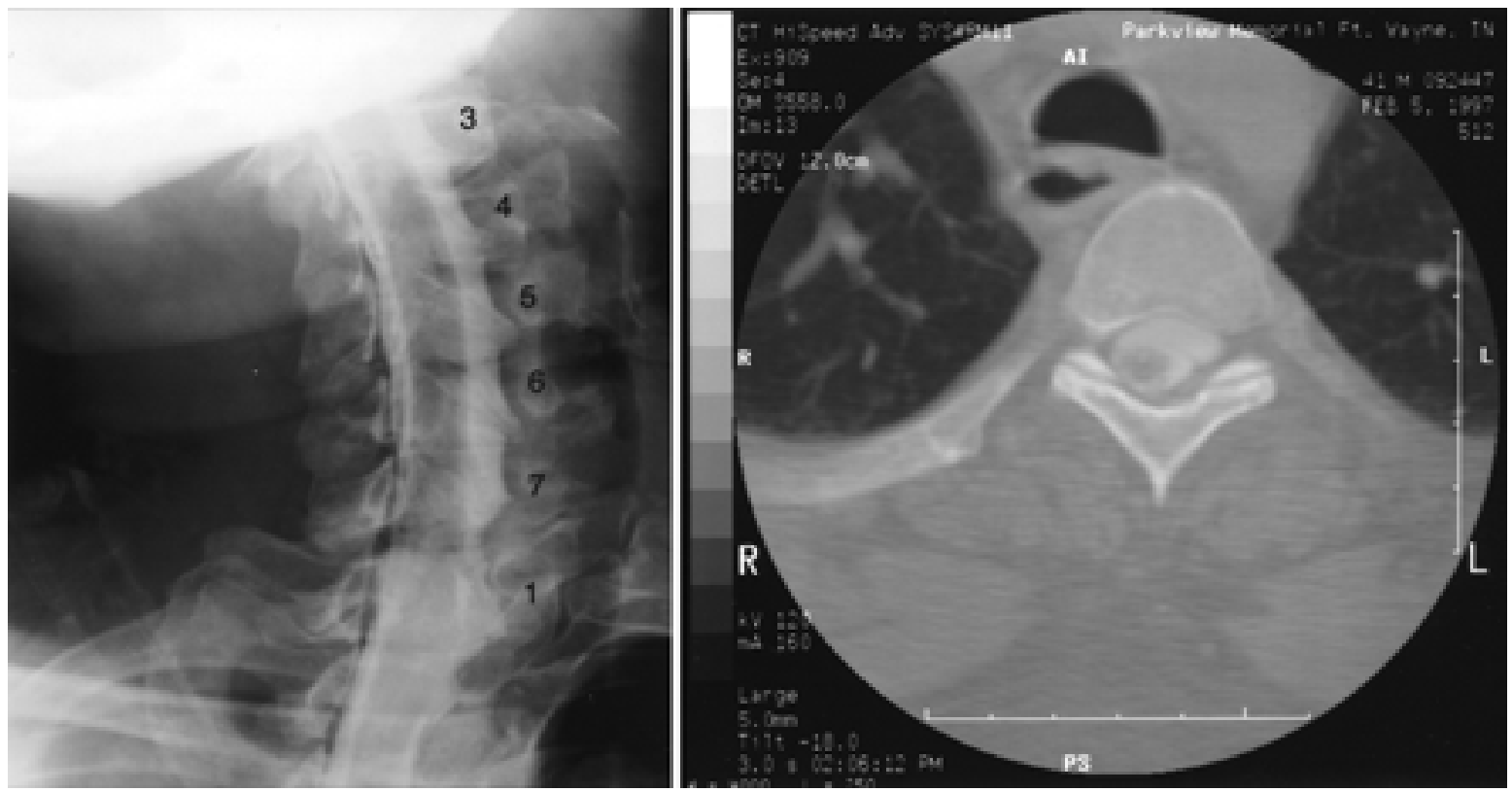

Fig. 2. Case 1. Left and Right: Cervical myelograms revealing irregular left C-8 and T-1 nerve root sleeves (left) and a loculated anterior subdural fluid collection extending from C-7 to T-1 (right).

complained of hearing loss and decreased sense of taste and smell for the past 3 years. He was involved in a car accident 39 years earlier during which he suffered multiple traumatic injuries to his right upper extremity as well as a right BPI. His right arm was subsequently amputated at the transhumeral level. He underwent anticoagulation therapy with Coumadin for atrial fibrillation.

Examination. Physical examination was remarkable for end-gaze horizontal nystagmus to the left and sensori- neural hearing loss. The patient was unable to perform tandem walking and had exhibited a very unsteady gait. Brain and spinal MR imaging revealed hemosiderin deposits covering the entire meninges. Cervical MR imaging also demonstrated a right $\mathrm{T}-1$ nerve root diverticulum. Cerebral angiography demonstrated no abnormality. This patient refused to undergo surgical exploration of the nerve root avulsion because of his advanced age, and further workup was not undertaken.
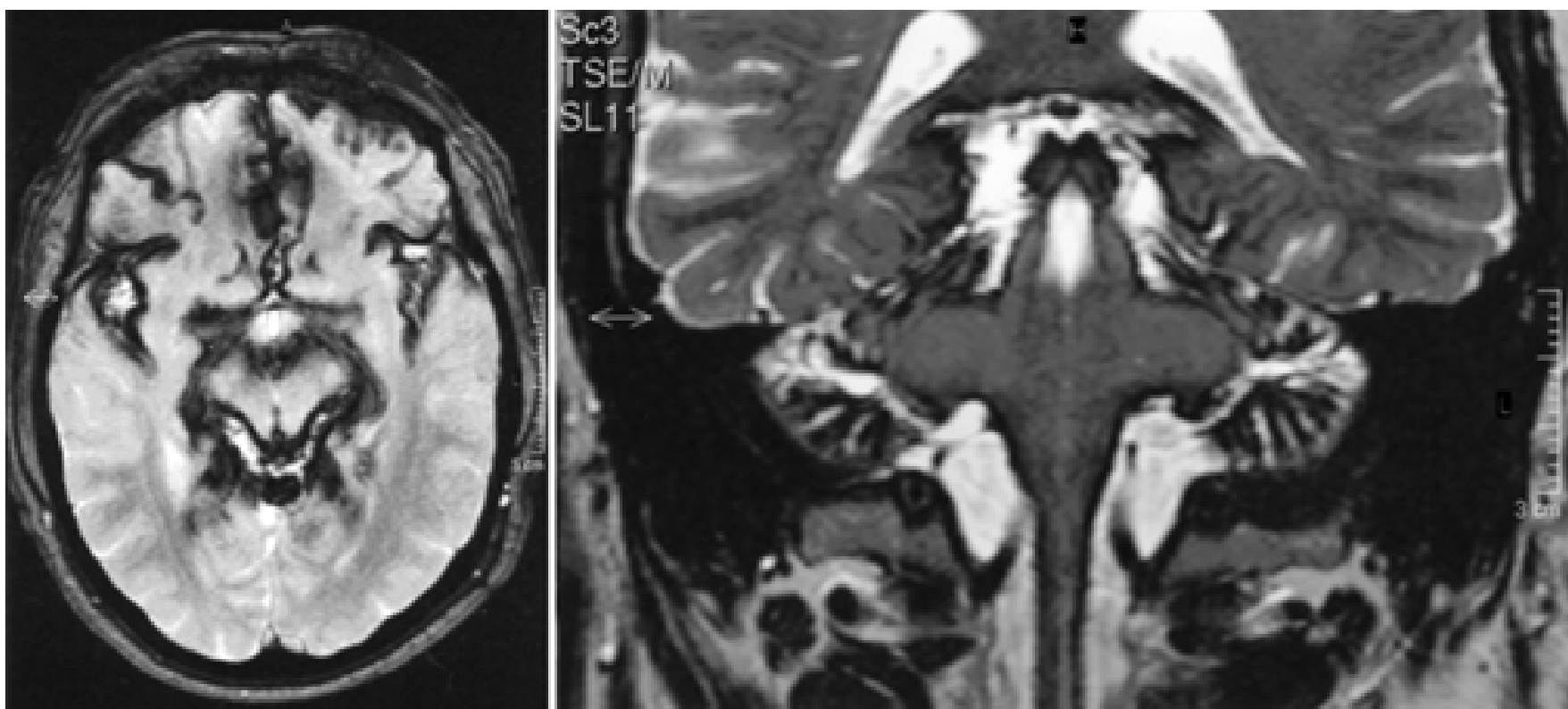

Fig. 3. Case 2. Left and Right: Axial proton density (left) and $\mathrm{T}_{2}$-weighted coronal (right) MR images revealing a rim of hypointensity around the sylvian fissures and cerebral convexities consistent with severe SS. Significant cerebellar atrophy is evident. 

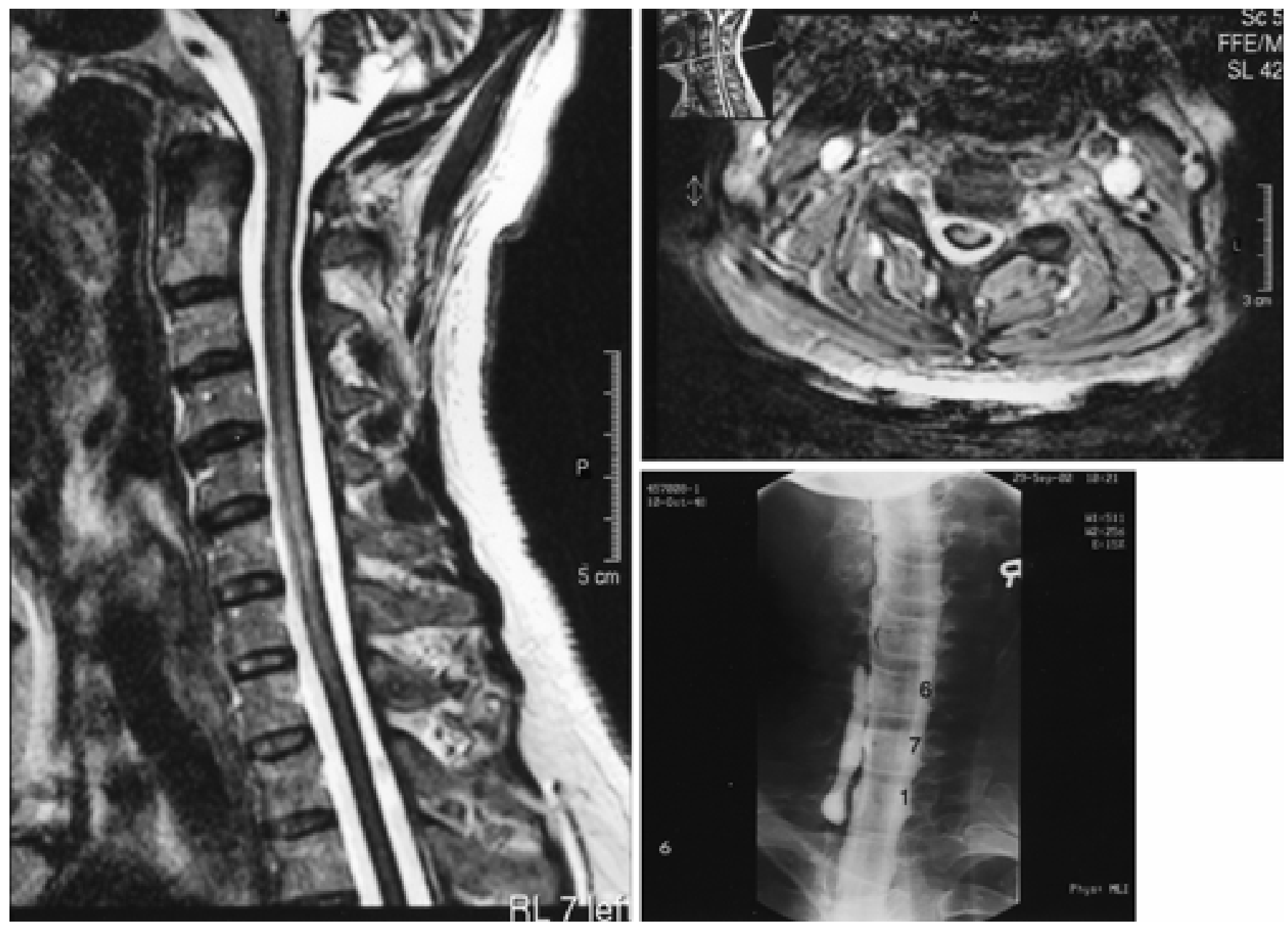

Fig. 4. Case 2. Left: Sagittal $\mathrm{T}_{2}$-weighted MR image demonstrating hemosiderin deposition along the spinal cord. The hyperintensity in the lower cervical spinal cord is consistent with a previously unrecognized concomitant old spinal cord injury. Upper Right: Axial $\mathrm{T}_{2}$-weighted MR image revealing an interruption of the rim of hypointensity around the spinal cord at the site of root avulsion. This finding was explained by the fact that the spinal cord pia mater was absent at the site of root avulsion. Hemosiderin deposition may therefore require an intact pial surface. Lower Right: Myelogram revealing left C7-T1 nerve root sleeve avulsions and pseudomeningoceles.

\section{DISCUSSION}

Surgeons treating BPIs should be aware of the potential for delayed neurological complications due to postinjury SS. Brachial plexus avulsion injury typically leads to significant injury of nerve root sleeves and subsequent pseudomeningocele or dural diverticulum formation. We believe that the associated dural tears may lead to scar formation with fragile vessels that could bleed intermittently, causing SS. ${ }^{1,12,18}$

Brachial plexus avulsion injury as a potential cause of SS is exceedingly rare; only seven cases have been reported in detail in the literature (Table 1). ${ }^{1,4,12-15,18}$ Indeed, a history of intradural surgery has been postulated as a risk factor for later development of SS. ${ }^{3}$ The long interval between BPI and the development of symptomatic SS in our patients are both consistent with previous reports in which a long presymptomatic phase was indicated. ${ }^{3}$ For these reported cases, the mean interval between the initial brachial avulsion injury and diagnosis of SS was 21 years, and the mean age at presentation was 49 years.
The diagnosis of central nervous system SS requires a high index of suspicion despite its characteristic MR imaging and CSF findings. Examination of CSF samples may reveal an increased number of RBCs with xanthochromia. Magnetic resonance imaging remains a reliable neurodiagnostic modality to identify a circumferential rim of hypointensity on the neural structures, indicative of hemosiderin deposition, on $\mathrm{T}_{2}$-weighted sequences. The greatest area of hypointensity is often on the superior vermis and the anterior portion of the cerebellar hemispheres.

The pathogenesis of SS remains poorly understood. If the excess intrathecal iron overloads the ferritin biosynthesis capacity of microglial cells, the resultant free radical damage, with subsequent lipid peroxidation, has been proposed as the responsible mechanism for neuronal injury. ${ }^{11}$ The peripheral nervous system is not involved in SS because central glia are affected by the disease process while Schwann cells are spared.

Limited treatment information is available concerning 
TABLE 1

Summary of details in patients with brachial plexus avulsion injury and delayed SS*

\begin{tabular}{|c|c|c|c|c|c|}
\hline Authors & $\begin{array}{l}\text { Age (yrs), } \\
\text { Sex }\end{array}$ & $\begin{array}{l}\text { Interval From } \\
\text { Injury to } \\
\text { Symptoms (yrs) }\end{array}$ & $\begin{array}{l}\text { Root } \\
\text { Avulsion } \\
\text { Level(s) }\end{array}$ & $\begin{array}{l}\text { Presenting Sign } \\
\text { \& Symptoms }\end{array}$ & Intervention \\
\hline Lewey \& Govons, 1942 & $36, \mathrm{M}$ & 15 & NS & $\begin{array}{l}\text { hearing loss, ataxia, urinary } \\
\text { \& speech difficulties }\end{array}$ & $\begin{array}{l}\text { posterior fossa exploration: } \\
\text { hemosiderin-stained } \\
\text { cerebellum }\end{array}$ \\
\hline $\begin{array}{l}\text { Bracchi, et al., } 1993 \\
\text { Fishman, } 1993 \dagger\end{array}$ & 47, M & 17 & C-8 & anosmia, hypotonia, ataxia & none \\
\hline Bonito, et al., 1994 & $51, \mathrm{M}$ & 15 & C-8 & $\begin{array}{l}\text { progressive ataxia \& } \\
\quad \text { genitourinary dysfunction }\end{array}$ & $\begin{array}{l}\text { repair of pseudomeningo- } \\
\text { cele, coagulation of scar }\end{array}$ \\
\hline Tapscott, et al., 1996 & $46, \mathrm{M}$ & 16 & $\mathrm{C} 5-\mathrm{T} 1$ & $\begin{array}{l}\text { tinnitus, sensorineural } \\
\text { hearing loss, imbalance }\end{array}$ & $\begin{array}{l}\text { exploration of C-5 pseudo- } \\
\text { meningocele; large vein } \\
\text { in the wall cauterized }\end{array}$ \\
\hline Maggioni, et al., 1997 & $51, \mathrm{M}$ & 21 & NS & hearing loss, ataxia & none \\
\hline Konitsiotis, et al., 2002 & $45, \mathrm{M}$ & 19 & $\begin{array}{c}\text { cervical } \\
\text { roots }\end{array}$ & $\begin{array}{l}\text { hearing loss, ataxia, } \\
\text { paraparesis, \& headaches }\end{array}$ & none \\
\hline \multirow{4}{*}{$\begin{array}{l}\text { Kole, et al., } 2004 \\
\text { present cases }\end{array}$} & $51, \mathrm{M}$ & 22 & $\mathrm{C} 8-\mathrm{T} 1$ & hearing loss, ataxia & epidural vein coagulated \\
\hline & $42, \mathrm{M}$ & 13 & $\begin{array}{c}\mathrm{C}-7 \& \\
\mathrm{~T}-1\end{array}$ & $\begin{array}{l}\text { hearing loss, ataxia, } \\
\text { paraparesis, memory } \\
\text { difficulty }\end{array}$ & $\begin{array}{l}\text { coagulation of abnormal } \\
\text { vessels in pseudo- } \\
\text { meningocele }\end{array}$ \\
\hline & $52, \mathrm{M}$ & 37 & $\mathrm{C} 7-\mathrm{T} 1$ & hearing loss, ataxia & $\begin{array}{l}\text { resection and ligation of } \\
\text { pseudomeningoceles }\end{array}$ \\
\hline & $69, \mathrm{M}$ & 36 & $\mathrm{~T}-1$ & $\begin{array}{l}\text { hearing loss, ataxia, } \\
\text { decreased sense of taste } \\
\text { \& smell }\end{array}$ & none \\
\hline
\end{tabular}

$* \mathrm{NS}=$ not stated.

$\dagger$ No details were provided for the two patients in this report.

patients in whom SS develops after BPIs. The authors of three separate case reports have advocated exploration of the pseudomeningoceles caused by cervical root avulsion. ${ }^{1,12,18}$ Friable vessels in the scarred wall of the pseudomeningocele, similar to our findings in Case 1, were exposed and coagulated. In one of these patients CSF studies demonstrated no further subarachnoid bleeding 4 weeks postoperatively, whereas in the other two patients the abnormal increase in the number of RBCs persisted at the 8-month and 2.5-year follow up but declined from the preoperative levels. These follow-up periods were too short to allow adequate assessment of surgery-related outcomes.

Long-term outcome data in patients who presumably have undergone surgical closure of the leakage are unavailable. These data are important in gauging the efficacy of surgical treatments and developing future therapies. Outcomes, however, are difficult to evaluate based on CSF profiles in patients who have presumably undergone repair of the bleeding source, because intrathecal bleeding is sporadic and in as many as $80 \%$ of patients with untreated SS a normal CSF profile without RBCs may be demonstrated. ${ }^{15}$ Because the hemosiderin deposits, secondary to preoperative bleeds, may continue to injure neuronal tissues, prevention of further hemorrhage may not make a significant difference in the overall clinical outcomes. Because SS is associated with a disabling and progressive course, thorough imaging evaluation of the entire neuraxis is warranted to locate any potential bleeding source. Iron chelation therapy has been used in a small number of patients without significant success, and no effective medical treatment is available at this time. ${ }^{3}$
Surgical exploration of the potential bleeding site remains a reasonable option.

\section{CONCLUSIONS}

Traumatic pseudomeningoceles developing after brachial plexus avulsion injury may be the source of intrathecal bleeding in patients with SS. At this time, the bleeding may be too slow and infrequent to allow current imaging modalities to localize the source accurately. Repair and closure of these dural defects caused by root avulsion injury may be a reasonable approach to prevent further intrathecal bleeding and additional neuronal injury. Long-term outcome evaluation is needed to determine the efficacy of surgical treatment in this subgroup of patients. Targeted diagnostic tools may lead to the development of reliable techniques for elucidating the site of hemorrhage in patients with an idiopathic form of SS.

\section{References}

1. Bonito V, Agostinis C, Ferraresi S, et al: Superficial siderosis of the central nervous system after brachial plexus injury. Case report. J Neurosurg 80:931-934, 1994

2. Bracchi M, Savoiardo M, Triulzi F, et al: Superficial siderosis of the CNS: MR diagnosis and clinical findings. AJNR 14: 227-236, 1993

3. Fearnley JM, Stevens JM, Rudge P: Superficial siderosis of the central nervous system. Brain 118:1051-1066, 1995

4. Fishman RA: Superficial siderosis. Ann Neurol 34:635-636, 1993

5. Gomori JM, Grossman RI, Bilaniuk LT, et al: High-field MR imaging of superficial siderosis of the central nervous system. J Comput Assist Tomogr 9:972-975, 1985 
6. Gomori JM, Grossman RI, Goldberg HI, et al: High-field spinecho MR imaging of superficial and subependymal siderosis secondary to neonatal intraventricular hemorrhage. Neuroradiology 29:339-342, 1987

7. Grunshaw ND, Blanshard KS, Hussain SS, et al: Superficial siderosis of the central nervous system - diagnosis by magnetic resonance imaging. Clin Radiol 48:186-188, 1993

8. Kale SU, Donaldson I, West RJ, et al: Superficial siderosis of the meninges and its otolaryngologic connection: a series of five patients. Otol Neurotol 24:90-95, 2003

9. Koeppen AH: Superficial siderosis of the central nervous system. J Neuropathol Exp Neurol 30:135-136, 1971

10. Koeppen AH, Barron KD: Superficial siderosis of the central nervous system. A histological, histochemical and chemical study. J Neuropathol Exp Neurol 30:448-469, 1971

11. Koeppen AH, Borke RC: Experimental superficial siderosis of the central nervous system. I. Morphological observations. J Neuropathol Exp Neurol 50:579-594, 1991

12. Kole MK, Steven D, Kirk A, et al: Superficial siderosis of the central nervous system from a bleeding pseudomeningocele. Case illustration. J Neurosurg 100:718, 2004

13. Konitsiotis S, Argyropoulou MI, Kosta P, et al: CNS siderosis after brachial plexus avulsion. Neurology 58:505, 2002
14. Lewey FH, Govons SR: Hemochromatotic pigmentation of the central nervous system. J Neuropathol Exp Neurol 1: 129-138, 1942

15. Maggioni F, Mantovan MC, Carollo C, et al: Morphological and functional study of a case of superficial siderosis of the central nervous system. Ital J Neurol Sci 18:113-118, 1997

16. O'Riordan JI, Javed M, McShane D, et al: Superficial siderosis of the central nervous system. Ir J Med Sci 165:182-184, 1996

17. Pyhtinen J, Paakko E, Ilkko E: Superficial siderosis in the central nervous system. Neuroradiology 37:127-128, 1995

18. Tapscott SJ, Eskridge J, Kliot M: Surgical management of superficial siderosis following cervical nerve root avulsion. Ann Neurol 40:936-940, 1996

19. Uchino A, Aibe H, Itoh H, et al: Superficial siderosis of the central nervous system. Its MRI manifestations. Clin Imaging 21: 241-245, 1997

Manuscript received March 23, 2004.

Accepted in final form April 2, 2004.

Address reprint requests to: Aaron A. Cohen-Gadol, M.D., Department of Neurologic Surgery, St. Marys Hospital, JO 1-229, 1216 Second Street SW, Rochester, Minnesota 55902. email: cohengadol.aaron@mayo.edu. 